\title{
Germanica
}

\section{Der beschädigte Eros}

Liebe, Erotik und Sexualität in den frühen Erzählungen Thomas Manns

L'Éros endommagé

The damaged Eros

\section{Karl Heinz Götze}

\section{OpenEdition \\ Journals}

Édition électronique

URL : http://journals.openedition.org/germanica/3738

DOI : ERREUR PDO dans /localdata/www-bin/Core/Core/Db/Db.class.php L.34 : SQLSTATE[HYO00]

[2006] MySQL server has gone away

ISSN : 2107-0784

Éditeur

Université de Lille

\section{Édition imprimée}

Date de publication : 30 juin 2017

Pagination : 117-133

ISBN : 9782913857391

ISSN : 0984-2632

Référence électronique

Karl Heinz Götze „Der beschädigte Eros“, Germanica [Online], 60 | 2017, Online erschienen am: 30 Juni 2019, abgerufen am 08 Januar 2021. URL: http://journals.openedition.org/germanica/3738 ; DOI: https://doi.org/10.4000/germanica.3738 


\title{
Der beschädigte Eros \\ Liebe, Erotik und Sexualität in den frühen Erzählungen Thomas Manns
}

\author{
Karl Heinz GöTZE \\ Université Aix-Marseille (AMU)
}

Wer die frühe Novellistik Thomas Manns bis hin zu Der Tod in Venedig auf dezidiert Gesellschaftliches befragt, muss sich mit einem ziemlich engen und starren Bild begnügen. Man erfährt wenig über die wilhelminische Gesellschaft, viel weniger als etwa bei Theodor Fontane, der, gestorben 1898, noch lebte, als Thomas Manns erster Novellenband bei S. Fischer erschien, obgleich wir geneigt sind, Fontanes Prosa einer ganz anderen literaturhistorischen Richtung und anderen Zeit zuzurechnen. Kaum Spuren auch von dem, was Heinrich Mann später als Geist der wilhelminischen Zeit zum Roman machte, Heinrich Mann, der ältere der beiden Brüder, mit dem Thomas, der jüngere, in der langen Periode seiner Anfänge eng kooperierte. Keine Spuren natürlich auch von den naturalistischen Blicken ins Leben der Schlesischen Weber durch Gerhard Hauptmann, den Thomas Mann später in Der Zauberberg als Mynheer Peeperkorn ironisch porträtieren sollte. Nichts über den preußischen Kulturkampf mit der katholischen Kirche, nichts über den Aufstieg der Sozialdemokratie, die das Bündnis von Bürgertum und Adel, das das zweite Reich regierte, zum Zittern und zum Zuschlagen trieb, nichts über den fatalen deutschen Griff zur Weltmacht, der die Zeit dem ersten Weltkrieg entgegen trieb und sich allenfalls als Schatten über manche der Texte legt. 
Das Personal der frühen Novellen Thomas Manns sind auf der einen Seite wohlhäbige Bürger und nicht allzu wohlhäbige Adelige, fern den unteren Klassen, aber auch fern der Macht und auf der anderen Seite bis zur Lächerlichkeit empfindsame Naturen, die sich manchmal nur für Künstler halten und manchmal wie Kröger oder Aschenbach auch welche sind. Dazwischen ist wenig, hin und wieder mal eine Dame vom Theater oder vom Ballett.

Vergleiche von Thomas Manns epischem Frühwerk mit Texten von Fontane oder von seinem Bruder Heinrich sind natürlich problematisch, selbst wenn sie nicht so gemeint sind, dass Thomas Mann hätte über andere Themen schreiben sollen. Fontane war alt, Thomas Mann sehr jung, als sich ihre Lebenszeit überschnitt. Fontane schrieb in Berlin, Thomas Mann in einer norddeutschen Hansestadt, dann in einer süddeutschen Residenzstadt und in Italien; der eine gab in realistischen Romanen Gesellschaftspanoramen, der andere erschrieb sich mit seinen Novellen, seinen Erzählungen die Voraussetzungen für die Buddenbrooks, die auf die Darstellung gesellschaftlicher Totalität zunächst durchaus nicht abzielten. Heinrich Mann war zu der Zeit, als sein Bruder mit Novellen die literarische Bühne betrat, noch keineswegs der scharfzüngige Kritiker der wilhelminischen Gesellschaft, der Der Untertan schrieb, sondern verfasste chauvinistisch-antisemitische Texte und orientierte sich an der französischen Décadence.

Der kursorische Blick auf Thomas Manns Themen und Personen mag aber erklären, warum der Zugang zu seinen frühen Texten für heutige Leser, heutige junge Leser vor allem, keineswegs einfach ist. Die zunächst in vielfältigen Formen immer erneut ins Auge fallende Problematik der (Un-)Vereinbarkeit bürgerlicher und künstlerischer Existenz, zwischen Geist und Leben, zwischen Bohème und ehrbarer Kaufmannsexistenz ist kein Zentralproblem heutigen Schriftstellerdaseins mehr und einer heutigen Bürgerexistenz schon längst nicht. Die Gesellschaftsstrukturen sind durchlässiger geworden, jedenfalls vielfältiger; Tabus sind verschwunden oder haben sich verlagert. Christian Kracht könnte zum Beispiel heute wohl kaum einen Roman darüber schreiben, dass sein Vater Generalbevollmächtigter beim Springer-Verlag war und er, der Sohn, zum Schriftsteller wurde. Wo, bitte, soll da das Problem sein? Im Gegenteil. Schließlich wissen wir nicht erst seit Bourdieus Les règles de l'art, dass ein ordentliches Erbe, das vom Literaturmarkt unabhängig macht, der literarischen Qualität häufig zu Gute kommt ${ }^{1}$.

1. - Pierre Bourdieu, Les règles de l'art. Genèse et structure du champ littéraire, Paris, Seuil, 1992. 


\section{Liebe und Erotik: Das dynamische Element in Thomas Manns frühen Erzählungen}

Hans R. Vaget, einer der besten Kenner und Kommentatoren der Erzählungen von Thomas Mann, hat zu Recht darauf hingewiesen, dass die starre Bürgerwelt, die sie zeigen, immer wieder durch Erotik und Sexualität aufgebrochen wird. Sie sind damit die wichtigsten Triebkräfte der Handlung, das wichtigste dynamische Element der Erzählungen:

Es kann [...] nicht verwundern, dass sich in der Welt dieses Autors [Thomas Mann, K.H.G.] Erotik und Sexualität als die letztlich alles beherrschenden Triebkräfte erweisen. [...] Thomas Manns Erzählungen, ungeachtet ihrer vielberufenen Künstler-BürgerThematik, die jedoch vordergründig bleibt, sind in einem eminenten Sinne erotische Erzählungen. [...] In diesem Werk ist ein Wissen um die Gefährdung der bürgerlichen Existenzformen gleichsam von unten durch die „Hunde im Souterrain“ -, das in der deutschen Literatur trotz WEDEKIND, HESSE und JAHNN einzigartig dasteht. Von den frühen Erzählungen bis zur letzten besiegelt Eros das Schicksal der Helden und Heldinnen; es gibt nur wenige Texte, in denen Geschlechtlichkeit keine Rolle spielt ${ }^{2}$.

Die Probe auf Vagets These ist anhand der Texte leicht gemacht. Schon der Schülerzeitungsaufsatz Vision, der sich im Sinne Hermann Bahrs etwas frühreif eine „minutiöse Gefühlsanalyse“3 vornimmt, handelt von Liebe, vielmehr von einer Liebesphantasie. Sie richtet sich aber nicht auf das Ganze einer geliebten Person, sondern allein auf „,die Mädchenhand“. Übrigens „erlischt“ die Liebe ,„äh“ wie die Perle aus dem Kelch, als sie an die Oberfläche steigt. Was bleibt, ist die ebenso weinerliche wie narzisstische Selbstversicherung: „Du liebtest mich doch“4. In der Sprache der Psychoanalyse würde man hier Fetischismus (der Teil für das Ganze) und Narzissmus konstatieren.

Gefallen beschreibt in klassischer Novellenfiguration die Enttäuschung junger Herren, die die angebetete Dame vom Theater zunächst zur „Heilige[n]“" stilisieren $(F E, 46)$, um dann die Doch-nichtso-ganz-Heilige enttäuscht als Hure zu schelten: „Wenn eine Frau heute aus Liebe fällt, so fällt sie morgen um Geld.“ $(F E, 49)$ Bei Peter Gay lässt sich nachlesen, dass das ausgehende 19. Jahrhundert gera-

2. - Hans R. Vaget, „Die Erzählungen“, in: Helmut Koopmann (Hrsg.), ThomasMann-Handbuch, 3. Aufl., Stuttgart, Kröner, 2001, S. 541.

3. - Ebd., S. 553.

4. - Thomas Mann, Frühe Erzählungen 1893-1912. In der Fassung der Großen kommentierten Frankfurter Ausgabe, 2. Aufl., Frankfurt a.M., Fischer Taschenbuch Verlag, 2014, S. 13. Im Folgenden abgekürzt durch die Sigle $F E$ und Seitenangaben in Klammern im Text. 
dezu besessen war vom Phänomen der Prostitution, einer „Realität, um welche sich dichte Nebelschwaden der Phantasie brauten." ${ }^{5}$ Eine Realität unzweifelhaft. Ein Wissenschaftler wie Bracebridge schätzte 1862 die Zahl der Londoner Prostituieren auf 80 000, Maxime du Camp zehn Jahre später die Zahl der käuflichen Mädchen in Paris auf sage und schreibe $120000^{6}$. Und an Phantasie hat es auch nicht gefehlt, wenn man an Maupassants Geschichte von den frommen Dirnen in der Maison Tellier oder der der patriotischen Hure in Boule de suif, an Huysmans' Marthe, an Edmond de Goncourts La fille Eliza, an Verdis La Traviata oder die zahlreichen „Süßen Mädel“ in Schnitzlers Werk denkt. Die Prostituierten nahmen, aber vor allem zahlten auch den Preis für die Spaltung zwischen der Idee bürgerlich-reiner, ehefähiger Weiblichkeit einerseits, enthemmter Wollust andererseits in den Köpfen ihrer Kunden. Thomas Mann verfügt, als er Gefallen schreibt, noch nicht über die Ironie, das Fragwürdige im Frauenbild der männlichen Akademikerrunde zum Vorschein zu bringen.

\section{Arten, Abarten und Abwesenheiten der Liebe in den frühen Erzählungen}

Manns frühe Novellen lesen sich an manchen Stellen wie ein narrativ illustriertes Kompendium sexueller Abnormitäten. Ein solches Kompendium hatte 1896 der Psychiater und Gerichtsmediziner Richard von Krafft-Ebing vorgelegt. Das war ein erheblicher Schritt in Richtung auf die wissenschaftliche Klassifizierung (und gesellschaftliche Diskriminierung) dessen, was der Zeit als pervers galt und, häufig seit der Antike beschrieben, am Ende des 19. Jahrhunderts erstmals definiert und mit einem Namen versehen wurde. So ,erfand“ der ungarische Arzt Karoly Maria Benkert 1869 den Begriff der „Homosexualität“, Krafft-Ebing führte in seiner Psychopatia Sexualis ${ }^{7}$ die Begriffe des „Sadismus“ und „Masochismus“ ein. Derlei Begriffe wurden also nicht von Freud „erfunden“ und von Mann direkt rezipiert, wie die ziemlich exakte zeitliche Übereinstimmung zwischen der Entstehung von Manns Novellen der ersten Vorkriegszeit und der Entwicklung der theoretischen Grundlagen der psychoanalytischen Theorie glauben machen will. Thomas Mann hat Freud gründlich erst in der Mitte der zwanziger Jahre gelesen und in sein Weltbild eingebaut.

5. - Peter Gay, Die zarte Leidenschaft. Liebe im bürgerlichen Zeitalter, Berlin, Siedler, 1999, S. 354.

6. - Ebd., S. 359.

7. - Richard von Krafft-Ebing, Psychopathia Sexualis, Stuttgart, Ferdinand Enke Verlag, 1886. 
Dies vorausgeschickt, kann man vielen Personen der in Frage stehenden Erzählungen ihre Perversionen auf den Kopf zusagen. Sadismus findet sich vielerorts, etwa in dem grausamen Spiel der Verführung, das Gerda von Rinnlingen mit dem verwachsenen kleinen Herrn Friedemann treibt, bis er ,,behandelt von ihr wie ein Hund, am Boden lag“ gepackt von einer ,,irrsinnige[n] Wut [...], die er bethätigen musste, sei es auch gegen sich selbst" $(F E, 118)$ und sich ins Wasser fallen lässt. Narzisstische Wut, die keinen Ausweg hat, schlägt um in den definitiven masochistischen Akt.

Eine ähnliche Deformation sehen wir in der Geschichte von Tobias Mindernickel (FE, 181-193), von allen verlacht und verachtet, dessen aufopfernde Liebe zu seinem hilflosen und hilfsbedürftigen Hund im Moment von dessen Genesung und Emanzipation umschlägt in wütende Mordlust. Die Liebe zum leidenden Geschöpf entpuppt sich als ärmlicher Besitzwunsch und perverse Selbstliebe.

Grotesker noch finden sich sadistische Bosheit einerseits, demüitige Selbstverachtung andererseits in Luischen (FE, 160-181). Eine schöne Frau, Amra Jacoby, ist verheiratet mit einem unansehnlichen, fetten, unterwürfigen Rechtsanwalt, den sie vor aller Augen mit einem jungen, mäßig begabten Musiker betrügt. Damit nicht genug, zwingt sie ihren hörigen Gatten bei einer von ihr organisierten, von ihm bezahlten Soiree nach einer Komposition ihres Liebhabers, „Luischen“ betitelt, im rosa Röckchen auf der Bühne zu tanzen, bis er tot umfällt - eine Geschichte über Sadismus, Hörigkeit und Ehe zugleich. Auch die phantastische Geschichte Der Kleiderschrank (FE, 193-204), in der die sich leidenschaftlich Küssenden sich zugleich Messer in den Rücken stechen, verknüpft Liebe unauflöslich mit Aggression und mit Schmerz.

Wälsungenblut (FE, 429-464), die einzige dieser Geschichten, die Skandal gemacht hat, zeigt vor dem Hintergrund des Wagnerschen Mythos den Inzest eines radikalästhetischen Geschwisterpaares, dessen Motive freilich nicht ödipal bestimmt sind, sondern eine Art dekadentes Experiment gegen die Trivialität des (verwöhnten) Daseins bilden.

Homosexualität findet sich nicht erst in Der Tod in Venedig (FE, 501-593), sondern in jugendlich-harmloser Beimischung schon in Wie Jappe und Do Escobar sich prügelten (FE, 482-501). Im letzteren Text wird sie handgreiflich, im ersten ist sie mit Händen zu greifen, obgleich Aschenbach bis zum Tode die Hände zur Faust ballt, um ihnen ihren Willen nicht zu lassen, was ihm am Ende freilich auch nichts nützt. Ein Glück (FE, 381-396) erzählt von der plötzlich aufkeimenden Liebe zwischen zwei Frauen aus zwei gründlich verschiedenen Gesellschaftsschichten. Die eine ist Tänzerin und wird nach einem Auftritt ihrer Truppe vom Herrn Baron, dem wilhelminischen Offizier, auf grobe, die Gefühle der Baronin verletzende Weise öffentlich umworben. Schließlich wendet sich die Umworbene gegen den Baron, 
der so gerne Rittmeister wäre, entschuldigt sich bei der Baronin, und für einen kurzen Moment ist etwas zwischen den beiden Frauen, was in der Ehe der Baronin nicht mehr ist, vielleicht niemals war: „Denn ein Glück, ein kleiner Schauer und Rausch von Glück berührt das Herz, wenn jene zwei Welten, zwischen denen die Sehnsucht hin und wieder irrt, sich in einer kurzen, trügerischen Annäherung zusammen finden.“ (FE, 395)

Die Liste unserer Hinweise auf Elemente der Psychopatia Sexualis in Thomas Manns Novellen ließe sich ohne Not noch entschieden verlängern. Dass damals die Homosexualität, die weibliche wie die männliche, zu den sexuellen Abnormitäten gerechnet wurde, muss kaum erinnert werden. Wer sie in Deutschland praktizierte und dabei ertappt wurde, verlor nicht nur seine bürgerliche Ehre, sondern wurde strafrechtlich verfolgt, wenngleich sie, wie etwa das Schicksal Fürst Philipp zu Eulenburgs zeigte, auch in der Hofkamarilla Wilhelms II. verbreitet war und sogar den mächtigsten deutschen Waffenschmied antrieb, Friedrich Krupp. Der spektakuläre Prozess gegen Oscar Wilde im Jahre 1895 beschäftigte nicht nur das literarische Europa, sondern die Massenpresse in England wie auf dem Kontinent. Unbescholtenheit vor der Ehe, Monogamie, Geschlechtsverkehr in „vernünftigen“ Maßen mit Willen zum Kind und Verzicht auf alle sexuellen Abnormitäten das war der herrschende bürgerliche Diskurs in allen Fragen von Erotik und Sexualität. Selbst Freud, vertraut mit der enormen Vielfalt sexueller Praktiken und Verbote, hielt normativ daran fest, dass alles, was nicht auf das Sexualziel gegengeschlechtlicher genitaler Vereinigung gerichtet sei, als Abweichung betrachtet werden müsse.

Statt weiter auszugreifen in der Besichtigung von Manns weit gespannter Phänomenologie der Sexualität, empfiehlt sich die Gegenprobe: In welcher der 25 Novellen bis hin zu Der Tod in Venedig spielen Liebe und Erotik eigentlich keine Rolle?

Thomas Mann selbst hat Der Tod (FE, 71-79), einen Kurztext in Tagebuchform, im Jahre 1896 zu einem Preisausschreiben der Zeitschrift Simplizissimus eingereicht. Teilnahmebedingung war, dass im eingereichten Text „die ,sexuelle Liebe' keine Rolle spielt.“8 Der Tod erfüllt in gewisser Weise diese Bedingung, denn eine Frau kommt nicht vor, auch kein homosexueller Partner, sondern nur der Tagebuchschreiber, in seinem einsamen Haus auf den Tod wartend, sein Diener, der Arzt und seine zwölfjährige Tochter Asuncion, die überraschend zuerst vom Tod geholt wird. Liest man genauer, so merkt man, dass die immer wieder als „klein“ und in Diminutiven bezeichnete Tochter immerhin zwölf

8. - Thomas Mann, Briefe an Otto Grautoff 1894-1901 und Ida Boy-Ende 19031928, Peter de Mendelssohn (Hrsg.), Frankfurt a.M., S. Fischer, 1975, S. 78. Brief an Grautoff vom 27.9.1896. 
Jahre alt ist: „Habe ich selbst den Tod an Dein Bettchen gezogen, habe ich Dich getötet, meine kleine Asuncion? Ach, das sind grobe, armselige Worte für feine und geheimnisvolle Dinge!“ $(F E, 78)$ Nein, sexuelle Liebe spielt hier keine Rolle, aber der Text ist getränkt von einer schwülen, verdrängten, inzestuös gefärbten Erotik.

Ganz ohne Sexualität und Erotik sind nur zwei Texte, Tobias Mindernickel und Der Weg zum Friedhof. Aber selbst für Tobias Mindernickel, so haben wir schon gesehen, gilt das nur mit Einschränkung. Sicher, in seiner Welt ist keine Frau, er ist zu gedrückt, um ein Weib auch nur aus der Ferne zu begehren, aber er findet ein Ersatzobjekt für seine Fürsorge wie für seine Mordlust: Hund statt Frau, aber immerhin Begehren.

Bleibt eigentlich nur Lobgott Piepsam, der arme Held von Der Weg zum Friedhof. Piepsam ist ,verwitwet, verwaist und von aller Welt verlassen; er hatte nicht eine liebende Seele auf Erden.“ (FE, 213) Zudem ist er arbeitslos, Trinker und ordnungsliebend bis zum Umfallen.

Piepsam und Mindernickel haben eine Gemeinsamkeit, die schon ihren Namen eingeschrieben ist. Vögel, die nicht singen können, piepen. Jemand der „zum Piepen“ ist, ist lächerlich. Wenn es „,bei ihm piept“, dann ist er nicht recht bei Verstand. „Nickel“ ist ein Metall oder ein Geldstück geringen Werts, bei unserem Tobias weiter gemindert durch die Vorsilbe seines Namens. Es dürfte kaum ein Zufall sein, dass dies unter den Mittelpunktfiguren von Thomas Manns frühen Novellen die beiden einzigen geringen Standes sind. Es fehlt ihnen an fast allem, so auch an Frauen, an Erotik und längst schon an Sexualität. Die Überzeugung, dass verfeinerte Erotik, so bizarr sie auch ist, nur den höheren Schichten der Gesellschaft möglich sei, gehört am Ende des 19. Jahrhunderts zu den verbreiteten Urteilen über den Zusammenhang von sozialer Stellung und Sexualität. „Es gibt eine Psychologie des gemeinen Mannes, die von der unsrigen ziemlich unterschieden ist" schrieb Freud 1883 an seine Braut. Die Brüder Goncourt waren sich sicher: „Viele Tugenden sind dem gemeinen Volk unmöglich. Unter zweitausend livres Jahreseinkommen existiert ein gewisses sittliches Empfinden nicht." 10 Und, so könnte man vermuten, eine breite Palette von Unsittlichkeit auch nicht.

\section{Liebe und Erotik vernichten}

Man sieht, die Liebe beim jungen Thomas Mann ist ein „weites Feld“", wie der alte Briest gerne formulierte, der alte Briest aus dem

9. - Zit. nach P. Gay, Leidenschaft, a.a.O., S. 394.

10. - Zit. ebd., S. 395. 
Roman, den Theodor Fontane 1894 dem Publikum vorlegte und den Namen von Briests unglücklicher Tochter trug. Seine eigentlich kreuzbrave, nur ein wenig naive Effi scheiterte an einer spät entdeckten, längst erledigten kleinen Liebesaffäre, die die Gesellschaft in ihrer grausamen Ehrbarkeit nicht verzeihen wollte. Die Geschichten, die Thomas Mann von der Liebe erzählt, gehen hingegen nicht mehr auf in dem Schema von Liebesverlangen und gesellschaftlicher Tabuierung. Freilich haben sie bei aller Vielfalt der Masken, die das Liebesbegehren aufsetzt, auch ein Grundmuster: Liebe ist nicht nur mit Aggression verschweißt (u.a. Der Kleiderschrank), sie hat nicht nur, wie der ,sonderbar[e] Herr“ auf dem Markusplatz in Venedig feststellt, Anteil an der ,große[n], allgemeine[n] Enttäuschung“ (FE, 79, 81), die für ihn das Leben ist, sondern sie vernichtet all diejenigen, die in ihren Sog geraten. Der Wille zum Glück, wie ihn die gleichnamige Erzählung darstellt (FE, 50-71), kann enorme Lebensenergie freisetzen, aber wo er an der Schwelle der Erfüllung ankommt, wartet der Tod.

Dieses Grundmuster zeigt sich am reinsten in der titelgebenden Novelle des ersten Novellenbandes, also in Der kleine Herr Friedemann sowie in der reifsten Erzählung der Vorkriegszeit, in Der Tod in Venedig. Der behinderte Herr Friedemann machte früh die Erfahrung, dass Liebesschwärmerei für ihn nur „Gram und Leid“ $(F E, 91)$ bringt. Er war bald fertig damit und lebte sein friedliches Leben, das er ,zärtlich liebte, das ihm sanft dahin floss, ohne große Affekte, aber erfüllt von einem stillen und zarten Glück, das er sich zu schaffen wusste.“ $(F E, 92)$ So lebte er dahin bis er im Theater, wie häufig bei Thomas Mann innerlich für Erschütterungen empfänglich gemacht durch die Musik Wagners ${ }^{11}$, betört wird durch die schöne, böse Gerda von Rinnlingen, Gattin des neuen Bezirkskommandeurs. Er erkennt seine Lebenszufriedenheit jenseits großer Affekte als Lüge und wirft sich, wissend darum, dass sie ihn quälen und verhöhnen will, in den Sog der femme fatale, ,überwältigt, in einem Zustand von Schwindel, Trunkenheit, Sehnsucht und Qual“" $(F E, 102)$ - bis zum bitteren Ende im nassen Grab des Flusses.

Gustav Aschenbach ist nicht behindert. Gustav Aschenbach ist auch nicht klein, sondern der repräsentative Schriftsteller seines Landes. Sein Verhältnis zur Kunst beschränkt sich nicht auf Lohengrin-Aufführungen in der Provinz. Sein Leben ist Kunst, er konstruiert sich selbst nach

11. - Vgl. Andreas Bloedorn, „Liebe und Erotik im Werk Thomas Manns“, in: Andreas Bloedorn und Friedhelm Marx (Hrsg.), Thomas Mann-Handbuch. Leben Werk - Wirkung, Stuttgart und Weimar, J.B Metzler, 2015, S. 320-322. Daselbst weitere Literaturhinweise zum Thema. Siehe auch Dietmar Krug, Eros im Dreigestirn. Zur Gestaltung des Erotischen im Frühwerk Thomas Manns, Frankfurt a.M. u.a., Peter Lang, 1997, S. 22 ff. 
dem Modell der Neuklassik, zwingt sich zu Stil, Form und Moral. Aber auch in sein Leben bricht in Venedig die unwiderstehliche erotische Leidenschaft, an der er sehenden Auges zu Grunde geht.

Dieses Grundmuster des Liebesleids hatte vor Aschenbach schon Klaus Heinrich (Königliche Hoheit) erlebt, auf Hans Castorf und Joachim Ziemßen (Der Zauberberg), den Joseph aus dem Joseph-Roman, Adrian Leverkühn (Doktor Faustus) und Frau von Tümmler (Die Betrogene) wartete es noch. Sie alle werden im geistreich ersonnenen und kunstvoll befestigten Bau ihrer Existenz vom Eros geschlagen und vernichtet.

Es ist vielfach darauf hingewiesen worden, welchen intellektuellen Anregungen sich Thomas Manns Auffassungen von Liebe und Erotik verdanken ${ }^{12}$. Man darf sich solche Wirkungen nicht direkt und linear vorstellen, aber eben doch als höchst gewichtig und lang anhaltend, wenn auch immer wieder modifiziert. Zuvörderst ist die Philosophie Schopenhauers zu nennen, die bei der ersten Lektüre in den Jahren 1895/96 für den jungen Autor im bildsamen Alter ein Schockerlebnis gewesen ist. 1938 schreibt er selbst in seinem Schopenhauer-Essay: „Ich bezeuge, dass die organische Erschütterung, die er bedeutete [der ,Rausch' der Schopenhauer-Lektüre, K.H.G.], nur mit der verglichen werden kann, welche die erste Bekanntschaft mit der Liebe und dem Geschlecht in der jungen Seele erzeugt, - und dieser Vergleich ist nicht zufällig."13 Übernommen hat Thomas Mann von Schopenhauer den Gedanken vom Primat des Triebes gegenüber der Vernunft, der eine Grundgewissheit des abendländischen Denkens seit der Aufklärung in Zweifel zog. Der Intellekt, der sich Herr des Ich und Herr der Welt dünkte, erscheint ,sekundär und akzidentiell, als des Willens Diener und schwache Leuchte."14

Thomas Mann hat diesen Gedanken später bei Freud erneut gefunden, wenn auch anders formuliert und anders begründet. Er findet bei Freud die Vorstellung von der Dominanz des Triebes wieder und versucht sogar, Freuds Instanzenlehre, die sogenannte „Zweite Topik“ von 1923 (Das Ich und das Es) in die von Schopenhauer bekannten Begriffe rückzuübersetzen. Das „Es“, in dem Freud das Unbewusste ansiedelt, ist ihm der Bereich des Schopenhauerschen Willens, zentriert um das sexuelle Begehren, während das „Ich“ dem „Intellekt“ gleich gesetzt wird. Das ,Über-Ich“ wird hingegen vernachlässigt, sicher auch

12. - Neben Hans R. Vaget, „Die Erzählungen“, a.a.O., und A. Bloedorn, Liebe und Erotik, a.a.O., siehe u.a. Hermann Kurzke, Thomas Mann. Epoche, Werk, Wirkung, München, Beck, 1985, S. 112-118 und Borge Kristiansen, ,Thomas Mann und die Philosophie“, in: Helmut Koopmann (Hrsg.), Thomas-Mann-Handbuch, a.a.O., S. 259284.

13. - Thomas Mann, Werke. Das essayistische Werk, [= ders.: Schriften und Reden zur Literatur, Kunst und Philosophie, Bd. 2], Frankfurt a.M., S. Fischer, 1968, S. 276.

14. - T. Mann, „Freud und die Zukunft“, ebd., S. 217. 
wegen Manns bekannter Vorliebe für duale Konstruktionen, auf die noch zurückzukommen sein wird ${ }^{15}$. Längst vor Freuds zweiter Topik hat Thomas Mann die geglückte Formulierung von den „Hunde[n] im Souterrain"16 gefunden, die auf die tierischen, gefährlichen, eingesperrten Triebe verweisen, die unter der vernunftbeherrschten Bel Etage des bürgerlichen Hauses auf ihre Chance lauern wie das Heer der Angestellten auf die Umwälzung.

Der Wille, wie Schopenhauer ihn sieht, ,ist das Gegenteil des ruhenden Genügens, ist an sich selbst etwas fundamental Unseliges, er ist Unruhe, Streben nach etwas, Notdurft, Lechzen, Gier, Verlangen, Leiden und eine Welt des Willens kann nichts anderes als eine Welt des Leidens sein." 17 Es ist hier der Raum nicht, die Schopenhauerschen Konsequenzen der Willensverneinung und Todesmetaphysik darzustellen und noch weniger Thomas Manns komplexen Umgang damit, aber es bleibt doch allemal festzuhalten, dass der oben erwähnte Umschlag des Liebesdiskurses zwischen Fontanes Effi Briest und Thomas Manns frühen Erzählungen ohne den Einfluss Schopenhauers nicht denkbar wäre. Dabei spielt keine Rolle, dass Schopenhauers Hauptwerk Die Welt als Wille und Vorstellung, auf dessen 44. Kapitel mit dem Titel Metaphysik der Geschlechtsliebe sich Thomas Mann vor allem bezieht, schon 1819 erschienen und im Zeitalter der Dominanz Hegels lange Zeit weitgehend unbeachtet geblieben war. Schopenhauers Breitenwirkung setzt erst lange nach seinem Tode (1860) ein, wie auch der europäische Nietzsche-Kult erst seine ungeheure Dynamik gewinnt, als der Philosoph geistig umnachtet dahindämmert.

Die Beschäftigung mit Nietzsche reißt in Thomas Manns Leben nicht ab. In unserem Zusammenhang ist der Bezug auf Nietzsches Tragödienschrift ${ }^{18}$ von besonderer Bedeutung, also auf sein Erstlingswerk aus dem Jahre 1872. Auch hier findet, wie im Falle Schopenhauers, ein fundamentaler Bruch mit einem Grundtheorem der Goethezeit statt. Nietzsche bricht mit der von Winckelmann über Schiller und Schlegel bis Goethe reichenden Überzeugung, die griechische Antike sei eine heitere, glückliche, harmonische Welt gewesen. In seiner Sicht war die griechische Antike eine tragische Welt voller Konflikte, bestimmt durch die Mühsal des Daseins und dunklen Heroismus. Die Kunst, die daraus entsteht, oszilliert Nietzsche zufolge zwischen dem Charakter zweier

15. - Ebd., S. $218 \mathrm{f}$.

16. - T. Mann, Briefe an Otto Grautoff, a.a.O., S. 68. Brief vom 17.2.1896.

17. - T. Mann, ,Schopenhauer“, in: Ders., Schriften und Reden zur Literatur, Kunst und Philosophie, Bd. 2, a.a.O., S. 260.

18. - Friedrich Nietzsche, Die Geburt der Tragödie aus dem Geist der Musik, in: Giorgio Colli und Mazziono Montinari (Hrsg.), ders., Die Geburt der Tragödie aus dem Geist der Musik, Unzeitgemäße Betrachtungen I-IV, Nachgelassene Schriften 1870-1873, München, dtv, 1999. 
Gottheiten, Apollon und Dionysos. Das Apollinische bezeichnet die gestaltende, maßvolle, bändigende Kraft der Harmonie, das Dionysische ist demgegenüber die zerstörende, chaotische, ekstatische Kraft, die sich in der ungeregelten Feier, dem Exzess, der lustvoll-schrecklichen Orgie entlädt ${ }^{19}$. Der Dualismus von Apollinischem und Dionysischem wird zu Beginn des 20. Jahrhunderts, ausgehend von der Kunstgeschichte ${ }^{20}$ zum wohl einflussreichsten dualen Modell der deutschen Geistesgeschichte.

Leicht erkennt man die Grundstruktur des von Nietzsche entwikkelten Antagonismus in den oben analysierten Schriften wieder: Die vom Willen, vom Begehren der Sexualität Getriebenen verlassen ihre maßvoll, aber fest, sicher und harmonisch gegründete Existenz, weil sie dem Sog des Ekstatischen nicht widerstehen können, gleich ob sie Friedemann oder Aschenbach heißen, behindert oder repräsentative Dichterfiguren sind.

\section{Der Verzicht auf die Liebe ist Verzicht auf das Leben und die Kreativität}

Spätestens an dieser Stelle stellt sich unabweisbar die Frage, warum denn die beklagenswerten Helden der frühen Erzählungen von Thomas Mann nicht die Gefahren meiden, die so sichtbar von der Sehnsucht nach Geschlechtsliebe für Geist, Leib und Leben ausgehen. Eine ganze Reihe von Geschichten sind dieser Problematik gewidmet und werden vom Erzähler narrativ durchgespielt.

Die naheliegende, dem herrschenden Diskurs entsprechende Lösung wäre gewesen, überbordenden Liebesdrang an die Ehe zu knüpfen und damit sozialverträglich zu zähmen, nicht immer ganz apollinisch-schön gewiss, aber doch in sicherer Entfernung von den Gefahren dionysischer Selbst- und Zivilisationsvergessenheit. In den Geschichten, die hier Gegenstand sind, wird der Ehe eine solche salvatorische Funktion nirgends zugebilligt. Viele der erwähnten Geschichten sexueller Abartigkeiten werden ja von verheirateten Personen inszeniert oder unterlaufen ihnen. Ausdrücklich wird die Ehe zum Thema in Anekdote, die davon erzählt, wie die ,himmlische kleine Angela Becker“ zum „Ideal unserer Gesellschaft“ zu ihrem „Stern“, ihrem „Wunschbild“ wird und ,alt und jung in Liebesbande geschlagen“ hat. Bis dann in einem schrecklichen Moment ihr Gatte nicht mehr an sich halten kann und öffentlich ,,in furchtbarem Ausbruch das Bild einer Ehe, - seiner Hölle von einer Ehe [...]“ zum Besten gibt: Die Gattin, ,falsch, verlogen

19. - Vgl. Peter Sloterdijk, Der Denker auf der Bühne. Nietzsches Materialismus, Frankfurt a.M., Suhrkamp, 1986, [= edition suhrkamp Nr. 1553].

20. - Heinrich Wölfflin, Kunstgeschichtliche Grundbegriffe: Das Problem der Stilentwicklung in der neueren Kunst, München, Bruckmann, 1915. 
und tierisch grausam [...] wäscht sich ja nicht einmal! Sie ist träge dazu! Sie ist schmutzig unter ihrer Spitzenwäsche!“ (FE, 464, 465, 468)

In der Ehe also sucht der junge Thomas Mann den sicheren Schutz vor dem tödlichen Sog des Liebesbegehrens nicht. Ausdrücklich und vielfach versucht er es hingegen mit der Spaltung von Erotik und Sexualität, zwischen Gefühl und nacktem Trieb. „Ich sage, trennen wir den Unterleib von der Liebe“21, fordert Thomas Mann am 5. 3. 1895 in einem Brief an den befreundeten Journalisten Otto Grautoff. Noch deutlicher, auch in einem Brief an Grautoff vom November 1896: „Wie ich sie hasse, diese Geschlechtlichkeit [...], sie ist das Gift, das in aller Schönheit lauert!“22 Die Forschung konstatiert dann auch ziemlich durchgehend bei Thomas Mann eine tiefgehende Sexualfeindschaft ${ }^{23}$. Für die von uns untersuchten Texte gilt das allemal. Sie sind durchzogen von erotischer Sehnsucht, auch von sexuellen Phantasien, aber gelebte Sexualität spielt, wo überhaupt, allenfalls am Rande eine Rolle. Das gilt übrigens auch für den gleichzeitig entstehenden großen Roman, in dem von vielem die Rede ist, aber nicht von sexuellen Beziehungen zwischen den Paaren. Deren Beziehungen sind weitgehend von ökonomischen Überlegungen bestimmt. Tony Buddenbrook, die sich dagegen auflehnt, muss deshalb scheitern. Auch die Beziehung von Imma und Prinz Klaus Heinrich in Königliche Hoheit ist seltsam frei von Leidenschaft.

Vor den gefürchteten Capricen des Unterleibs schützt neben der Spaltung die Distanz, denn nur Kindern ist von Natur gegeben, was sich Das Wunderkind bei seinem Konzert wünscht (wenig kindlich ist sein Wunsch übrigens, hinter dem man den des Autors deutlich hervorlugen sieht): „Gibt es denn eine losgelöste Leidenschaft, eine Leidenschaft an sich und ohne irdischen Gegenstand, die nur ein inbrünstiges Kinderspiel wäre?“ ( $F E, 403)$ Ansonsten ist es sicherer, man belässt es wie der "Novellist" in Beim Propheten dabei, dem in Abwesenheit verehrten "Glücksfall von einem Geschöpf“" namens Sonja, leidend übrigens, vermittels ihrer Frau Mutter Grüße auszurichten und sogar, da das dem übervollen Herzen nicht reicht, am nächsten Morgen „,ungefrühstückt" $(F E, 414)$ ein „Blümchen“" zu senden.

So weit geht „Detlev“, der Held von Die Hungernden nicht, Künstler auch er, einsam auch er, auf Distanz begehrend auch er - begehrend Lilli mit den blauen Augen, die aber auf dem Fest, von dem erzählt wird, mit eben diesen blauen Augen einem „kleinen Maler“ tief in die seinen blickt, bis der wehmütig einsame Detlev ohne sie angesprochen zu haben gleichsam hungrig von dannen geht und sich am Ende auf der Straße als Genosse eines wüsten, verwilderten Arbeiters fühlt,

21. - T. Mann, Briefe an Otto Grautoff, a.a.O., Brief vom 5.3.1895.

22. - Ebd., 12.11.1896.

23. - Vgl. A. Bloedorn, „Liebe und Erotik“, a.a.O., S. 320 ff. 
der von außen neidisch auf das Fest der glücklich Besitzenden starrt. Schopenhauer muss herhalten, um für Detlev aus diesem Genossen am Ende noch einen Bruder im Geiste zu machen: „Ach, wir sind Alle Geschwister, wir Geschöpfe des friedlos leidenden Willens; und wir erkennen einander nicht.“ (FE, 380)

Der Figur des Bajazzo hat Thomas Mann überraschend viel von der eigenen Biographie geliehen. Der Bajazzo verfügt früh über die Fähigkeit zu gefallen, und eine nicht geringe Erbschaft versetzt ihn in die Lage, sich ganz den angenehmen und kultivierenden Dingen des Lebens zu widmen, ein ,ungestörtes und beschauliches Dasein“ am Ort seiner Wahl zu leben. „Ich schlenderte von einem Tage in den andern“ (FE, 135, 138), ohne Beruf, ohne Verpflichtungen, ohne gesellschaftlichen Verkehr. Man sieht kommen, dass dieses Leben, das sich kunstvoll einzureden versucht, glücklich zu sein, allmählich fad wird, und man sieht auf einem ,zweirädrigen Jagdwagen“ Abhilfe kommen: „Die Zügel hielt eine junge Dame von neunzehn, vielleicht oder zwanzig Jahren [...] von auserlesener Eleganz [...]" $(F E, 145,147)$. Die junge Dame, als Tochter eines Justizrats zur guten Gesellschaft der Stadt gehörend, verlobt sich schließlich mit dem schmucken Assessor Dr. Witznagel, während der früher so gefällige und eloquente Bajazzo bei der einzigen Begegnung mit der heimlich Begehrten eine unglückliche Figur macht. „Was geschah? Ah, nichts!“ (FE, 154)

Es ist unübersehbar, dass die angstvolle Distanznahme zum Liebesobjekt, ja zur Liebe überhaupt, die doch allein einen gewissen Schutz gegenüber ihrem zerstörerischen Sog zu versprechen scheint, einen hohen Preis kostet, den der Entfernung vom Leben und der Isolierung in einem schönen, kultivierten, geistigen, aber gänzlich luftleeren Raum. So schreibt Hermann Kurzke über den Bajazzo im Vergleich zum „Friedemann“: „Es widerfährt ihm [...] kein solcher Einbruch des Dionysischen, aber siehe da: das ist fast noch schlimmer. Er ist nicht einmal zu einem Selbstmord fähig." 24

Der Bajazzo, aber in unterschiedlichem Maße auch der „Novellist““ und ,Detlev“", der Ich-Erzähler von Enttäuschung, der Tagebuchschreiber von Der Tod, Albrecht van der Qualen von Der Kleiderschrank entsprechen dem Typus des „Dilettanten“, also dem Typus des Lebenszuschauers ohne wirkliche Kreativität und ohne Vitalität, in Schönheit erstarrt. Er wird gern als Schöpfung von Paul Bourget apostrophiert, dem er tatsächlich wichtige Züge verdankt, sollte jedoch eher als eine Art bürgerlicher Modetypus des ausgehenden 19. Jahrhunderts gesehen werden wie im 20. und 21. der „Yuppie“, der „Bobo“ oder im Moment der „Hipster“,

24. - H. Kurzke, Thomas Mann, a.a.O., S. 57. 
Typen, die sich heute freilich der künstlerischen Selbststilisierung weitgehend entschlagen haben.

Thomas Mann hat Paul Bourgets 1888 erschienene Physiologie de l'amour moderne zweifellos gelesen und von ihr wichtige Anregungen empfangen, wurde darüber hinaus auch indirekt durch Nietzsches Auseinandersetzung mit der Décadence auf ihn verwiesen. Die Physiologie Bourgets hat vor allem das Scheitern vielfältiger Liebesbeziehungen zum Thema und kann also auch in dieser Richtung als Vor-wurf zu Thomas Manns frühen Novellen gesehen werden. Entscheidend scheint mir in unserem Zusammenhang zu sein, dass Bourget trotz einer spürbaren Sympathie für den dilettantischen Lebensstil nicht etwa als einsinniger Propagandist des Dilettantismus gesehen werden darf, sondern der Décadence die Rückkehr zum Katholizismus verordnet. Das nun lag Thomas Mann fern, aber auch in seinen Erzählungen sind die Bajazzo-Typen nicht unbedingt ohne Sympathie gezeichnet, verfehlen aber allemal das Leben und die Liebe.

Das gelungenste Beispiel der Mannschen Kritik des Dilettantismus ist gewiss die Novelle Tristan, die in einem Schweizer Lungensanatorium angesiedelte Geschichte von Frau Gabriele Klöterjahns Verderben zwischen dem lebensfeindlichen Künstler Spinell sowie Vater und Sohn Klöterjahn, beide robust mit beiden Beinen im Leben stehend. Spinell ist bartlos, knabenhaft, der Wirklichkeit in keiner Weise zugetan, will sich nur der Kunst, der Schönheit und dem Tod widmen, obgleich er mit der Kunst so seine Schwierigkeiten hat, Schönheit nicht gerade ausstrahlt und den Tod nur anderen zu bringen versteht, in diesem Falle, indem er Frau Klöterjahn zum tödlichen Rausch bei der Darbietung des Tristan-Vorspiels drängt. Klöterjahn hingegen ist schon für den zutreffend charakterisiert, der weiß, dass „Klöten“ ein populärer norddeutscher Ausdruck für „Hoden“ ist.

Spinell ist natürlich auch in der Liebe nicht für das Grobe und Zupackende. Er mag Frauen nicht ansehen, sondern streift sie lieber ,im Vorübergehen mit einem halben Blicke“. „Aber der verwischte Schatten von ihr, den ich empfing, hat genügt, meine Phantasie anzuregen und mich ein Bild mit fortnehmen zu lassen, das schön ist [...] Gott, es ist schön!“‘ (FE, 335) Und zum Sterben schön ist dann Gabriele Klöterjahns Darbietung des Tristan-Vorspiels. In der folgenden Auseinandersetzung mit Klöterjahn hingegen macht Spinell eine schlechte Figur gegen Klöterjahns gesunden Menschenverstand: „Ich schiele den Weibern nicht am Gesicht vorbei, ich sehe sie mir an, und wenn sie mir gefallen, und wenn sie mich wollen, so nehme ich sie mir.“ $(F E, 365)$ Spinell kostet seine Neigung nicht das Leben, aber er wird schließlich von der pausbäckigen Gesundheit des Klöterjahn-Säuglings jämmerlich in die Flucht geschlagen. 
Thomas Mann vergisst nie, die Kreativität, vor allem die Kunstfertigkeit der kunstseligen Lebensverächter und Liebesvermeider ironisch in Frage zu stellen. Die Dilettanten sind keine wirklichen Künstler, sondern „künstlerische Menschen“, eine Unterscheidung, die noch in Heinrich Bölls Ansichten eines Clowns eine wichtige Rolle spielt. Wer die Liebe meidet, kann ebenso wenig Künstler sein wie der, der ihr ganz ihren Willen lässt oder ihn sich gänzlich zu eigen macht. Der Zusammenhang besteht nicht vor allem darin, dass Liebesabenteuer dem Künstler Stoff zutreiben, sondern ist energetisch gedacht, als Sublimierung von Triebenergie. Vom „Unterleib“ nämlich redete Thomas Mann nicht nur schlecht. Er schrieb ihm zugleich „eine ganze Menge Poesie“ 25 zu.

Die Liebe, die ganze Liebe, nicht die nach Spinells Art kastrierte, stellt die Energie zur Verfügung, mit der ein großes Werk überhaupt erst angetrieben werden kann. So ist übrigens auch die Bemerkung aus Schwere Stunde zu verstehen (,,ich darf nicht allzu sehr dein, nie ganz in dir glücklich sein, um dessentwillen, was meine Sendung ist...", $F E$, 428), die Schiller in den Mund gelegt wird, der nächtens zärtlich auf seine schummernde Gattin blickt.

Überblickt man die Rolle von Liebe und Erotik in den untersuchten Novellen, so lässt sich außer ihrer Omnipräsenz konstatieren, dass wir es bei aller Vielfalt der Varianten mit zwei grundlegenden Vorgangsfiguren zu tun haben: Entweder lässt sich das Personal auf die Liebe, auf das Begehren des Willens ein, erlebt dann bestenfalls einen dionysischen Rausch und geht zu Grunde, oder aber es meidet sorgsam die Nähe zum gefährlichen Sog der Erotik und verliert mit dem Verzicht auf die Liebe auch alle Lebensvitalität und Schöpferkraft. Mit Nietzsches Begriffen gesprochen: Entweder Auflösung im Dionysischen oder von allem Fleisch und Blut, von allem Leben gereinigte, also hohle Schönheit in apollinischer Gestalt, wobei gelingende Kunst ohne das dionysische Element nicht auskommt, ihm aber eine heroische Leistungsethik entgegensetzt, um nicht zu versinken.

\section{Die literarische Lösung eines unauflösbaren Dilemmas}

Theoretisch gesehen kommt Thomas Mann über das skizzierte Dilemma und den damit zusammenhängenden Dualismus nicht hinaus. Das galt auch noch, nachdem er die homoerotische Beziehung zu Paul Ehrenberg hinter sich gelassen hatte, nicht aber, wie spät die Tagebücher auswiesen, die Homosexualität ${ }^{26}$. Dass er sich 1904 durch die Heirat

25. - Zit. nach A. Bloedorn, „Liebe und Erotik“, a.a.O., S. 320.

26. - Dietmar Krug ist zuzustimmen, dass es die „1977 einsetzende Publikation der Tagebücher, die Thomas Manns homoerotische Neigungen ans Licht brachten“, 
mit Katia Pringsheim aus renommiertem, wohlhabendem Hause eine bürgerliche Verfassung gegeben hatte, änderte an der Grundstruktur seiner Sicht auf die Erotik wenig, wie noch die Schrift Über die Ehe ${ }^{27}$ aus dem Jahre 1925 zeigt, wo sich erneut Homosexualität und Ehe, Kunst und bürgerliches Leben, Radikalästhetik und Verantwortungsethik, Freiheit und Pflicht diametral gegenüber stehen, beide Seiten im Recht wie einst die Götter Apoll und Dionysos, aber auch auf ewig unverträglich. Diese Unverträglichkeit dürfte dem Werk Thomas Manns immer wieder Energien zugetragen haben.

Das Bemühen um Versöhnung der Sphären hört darum nicht auf und bringt noch im Scheitern bleibende Texte hervor, allen voran Tonio Kröger und Der Tod in Venedig. Tonio Kröger hätte die Geschichte des Bajazzo leben können, aber er nimmt seine Kunst in doppelter Weise in Zucht: indem er an ihr arbeitet und indem er ihr das Menschliche, Bürgerliche und Gewöhnliche als Gegengift gegen Dilettantismus, Bohèmewesen und Zigeunerhaftigkeit verordnet. In erotischer Hinsicht freilich will die Überwindung der Dekadenz in Richtung auf ein Leben nach dem Muster der früh vergeblich Geliebten mit Namen Hans und Inge, nicht gelingen. Kröger mit Hans R. Vaget ,geheime erotische Vitalität“" zuzuschreiben, die sich in der Fähigkeit zeige, „Hans und Inge gleichsam überall zu erblicken und so die erotischen Energien der Jugend, indem er sie auf andere, neue Konstellationen projiziert, zu erneuern“" 28 ist kaum zwingend. Der etwas gezwungenen Liebeserklärung Krögers ans Leben folgt jedenfalls keine an eine Frau, und alle Fragen des Unterleibs bleiben ungestellt und unbeantwortet.

In Tod in Venedig siegt das Abgründig-Dionysische über das (Neu-) Klassische in der Selbststilisierung Aschenbachs, siegt der Sog der erotischen Anziehung über die antrainierte literarische Leistungsethik, siegt die Homosexualität über die bürgerliche Wohlanständigkeit, ohne sie freilich zu provozieren oder gar zu verletzen. Nein, die Dekadenz, der Wille zur (Homo-)Sexualität, all das, was Kröger überwinden wollte, überwältigt Aschenbach und flutet dessen von langer Hand befestigte Deiche gegen die auflösende Kraft der Sinnlichkeit. „Im Tod in Venedig“, da ist Vaget zuzustimmen, ,wird die Homoerotik zugleich verworfen und gefeiert." 29 Nirgendwo sonst in Thomas Manns Erzählungen werden die beiden Grundtendenzen seiner Sicht auf den

gewesen ist, die die ,erotische Thematik [...] seit Ende der siebziger Jahre verstärkt ins Zentrum gerückt" hat. Dietmar Krug, Eros im Dreigestirn, a.a.O., S. 13.

27. - T. Mann, Schriften und Reden zu Literatur, Kunst und Philosophie, a.a.O.,

Bd. 1, Frankfurt a.M., 1968, S. 250-262.

28. - H. R. Vaget, „Die Erzählungen“, a.a.O., S. 566.

29. - Ebd., S. 581. 
Eros so eng geführt und so glücklich miteinander verwoben wie hier, glücklich für die Literatur, unglücklich für Aschenbach.

Was Thomas Manns Erotologie theoretisch nicht miteinander vermitteln kann, gelingt ihm in den besten Fällen mit den Mitteln des Schriftstellers, der sich Logik nicht abverlangen muss. Thomas Mann weiß das, ahnt es zumindest. Sonst fände man - ausgerechnet in den Betrachtungen eines Unpolitischen - nicht jene seltsame Passage über die erotische Ironie: „Der Geist, welcher liebt, ist nicht fanatisch, er ist geistreich [...] und sein Werben ist erotische Ironie [...] Ironie aber ist immer Ironie nach beiden Seiten hin; sie richtet sich gegen das Leben sowohl wie gegen den Geist [...]"30. Ironie muss nicht nach dem Rechten sehen und nicht nach dem Widerspruchsfreien, sie muss die literarischen Figuren nicht vor den Folgen ihres Handelns schützen. Sie bringt die Dinge ins Schwingen. Darin gleicht ihre Macht der der Erotik. 
